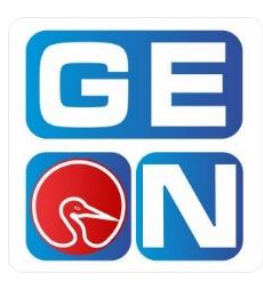

Revista GEON (Gestión, Organizaciones y Negocios.) ISSN: 2346-3910 en línea

revistageon@unillanos.edu.co

Universidad de los Llanos

Colombia

Pacheco Pérez, Camilo Ernestoi; González Jiménez, Guillermo Enrique; Mancera Ortiz, Andres Mauricio ${ }^{i i}$; Castro Riveros, Antonio Jose.

Seguridad y soberanía alimentaria en el departamento del Meta-Colombia: retos y oportunidades

Revista GEON, Vol. 5, No. 2, 2018

Pág. 82-95

Disponible en: https://doi.org/10.22579/23463910.9

${ }^{\mathrm{i}}$ https://orcid.org/0000-0002-3490-808X

ii https://orcid.org/0000-0002-1439-3269

Esta publicación se encuentra bajo licencia: Creative Commons

ReconocimientoNoComercialSinObraDerivada 4.0 Internacional
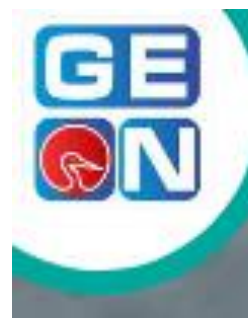

66

Se plantea la necesidad de ordenar el territorio de forma participativa y responsable anotando en torno ala seguridad y soberanía alimentaria

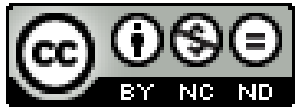




\title{
Seguridad y Soberanía Alimentaria en el Departamento del Meta . Colombia: Retos y Oportunidades
}

\section{Food Security and Sovereignty in the Department of Meta- Colombia: Challenges and Opportunities}

\author{
Camilo Ernesto Pacheco Pérez \\ Camilo.pacheco@campusucc.edu.co
}

Magister en Ciencias Económicas, Universidad Cooperativa de Colombia; Investigador delegado Red Investigadores del Meta REDIME línea seguridad alimentaria, Colombia

\section{Guillermo Enrique González Jiménez}

gugonzalez@uniminuto.edu

Especialista en Producción Agrícola Tropical Sostenible, Universidad Minuto de Dios; Investigador delegado Red Investigadores del Meta REDIME línea seguridad alimentaria, Colombia

\section{Andrés Mauricio Mancera Ortiz andres.mancera@unad.edu.co}

Especialista en Educación Cultura y Política, Universidad Nacional Abierta y a Distancia de Colombia; Investigador delegado Red Investigadores del Meta REDIME línea seguridad alimentaria, Colombia

\section{Antonio José Castro Riveros \\ ajcastro@unillanos.edu.co}

Magister en Administración con Especialidad en proyectos, Universidad de los Llanos, Grupo Investigación GYDO, Colombia

Revista GEON Vol. 5 No 2 julio-diciembre 2018

ISSN 2346-3910 en línea http://revistageon.unillanos.edu.co/index.php/geon/article/view/9 


\section{Resumen}

El trabajo en el campo evidentemente ha sido el pilar sobre el cual se ha fundamentado la economía departamental, y el desarrollo socio cultural de la región. En torno al sector agropecuario y el potencial productivo de cada zona, es que se han establecido asentamientos humanos en los diferentes municipios, se han trazado vías y se ha generado desarrollo que ha determinado en gran medida, los medios de subsistencia para las familias metenses. Por un lado, la cultura y el desarrollo de las comunidades históricamente ha estado ligada a la productividad de la tierra, a los servicios ecosistémicos que esta ofrece, y al potencial que ella representa en términos de biodiversidad. Por otro lado, la generación de recursos y de riqueza para la región evidentemente encontró y seguirá encontrando en el sector agropecuario, su musculo más fuerte, pues a través de la agricultura y la ganadería es que se han forjado grandes industrias que en la actualidad vienen trabajando en el territorio. La economía del departamento gira en torno al agro, encuentra que lo económico no solo incide en temas de generación de ingresos y egresos, o de importaciones o exportaciones, sino que está íntimamente ligado a dinámicas sociales como la agricultura familiar, la economía campesina y la seguridad y soberanía alimentaria, entre otros, esta última presente en los planes de ordenamiento territorial y en la agenda de grandes agencias de cooperación que hacen presencia en la región, pero que no han tenido resultados concretos que permitan sobre todo cumplir con el cometido de lo que significa ser un territorio soberano en términos de alimentación para sus comunidades..

Palabras claves: agroecología, economía campesina, agricultura familiar, sustentabilidad, alimentos, nutrición, Colombia.

\section{Abstract}

The work in the field has evidently been the pillar on which the departmental economy has been based, and the socio-cultural development of the region. Regarding the agricultural sector and the productive potential of each zone, human settlements have been established in the different municipalities, roads have been laid out and development has been generated that has largely determined the means of subsistence for the Metensian families. On the one hand, the culture and development of the communities has historically been linked to the productivity of the land, the ecosystem services it offers, and the potential that it represents in terms of biodiversity. On the other hand, the generation of resources and wealth for the region evidently found and will continue to find in the agricultural sector, its strongest muscle, because through agriculture and livestock is that they have forged large industries that currently come working in the 
territory. The economy of the department revolves around agriculture, finds that the economic not only affects issues of income generation and expenditures, or imports or exports, but is intimately linked to social dynamics such as family farming, peasant economy and agriculture. security and food sovereignty, among others, this last one present in the plans of territorial ordering and in the agenda of great cooperation agencies that are present in the region, but that have not had concrete results that allow above all to fulfill the task of what it means to be a sovereign territory in terms of food for their communities.

Keywords: agroecology, peasant economy, family agriculture, sustainability, food, nutrition, Colombia.

\section{Introducción}

El Departamento del Meta se encuentra ubicado en el centro del país, en la región de la Orinoquía colombina, y cuenta con $85.635 \mathrm{~km}^{2}$ lo cual le ubica como el cuarto departamento más grande del territorio nacional, extendiéndose desde el piedemonte de la cordillera oriental hasta las grandes sábanas de altillanura; cuenta con una gran biodiversidad y una excelente riqueza hídrica debido a sus grandes extensiones de paramo en lo alto de la cordillera, sus grandes caños, quebradas y ríos, y sus extensos humedales o morichales en las sabanas.

Su capital Villavicencio, se encuentra ubicada a tan solo 120 kilómetros de la ciudad de Bogotá, principal consumidor en el país de bienes y servicios, lo cual representa una gran oportunidad para la región en términos de desarrollo social, agroindustrial, turístico y económico, si se tiene en cuenta que el territorio se destaca principalmente por dos grandes actividades productivas, que entre otras son antagónicas y de cierta manera excluyentes por el impacto social que tiene la una sobre la otra.

La primera tiene que ver con la producción agropecuaria, en la que se incluyen actividades de monocultivos tanto agrícola como pecuaria; donde la ganadería, además de ser parte fundamental de la cultura llanera, por décadas ha sido la principal actividad del llanero metense; su arraigo por el ganado, el trabajo de llano y los grandes rodeos hacen del departamento uno de los principales proveedores de carne en el país. Por otro lado, la producción de petróleo en los últimos años ha estado jalonando la economía en la región; grandes compañías petroleras llegaron en las últimas décadas interviniendo a las comunidades en todo el territorio, evidenciándose como consecuencia la generación de drásticos cambios socioculturales, ambientales y productivos.

Lo anterior son aspectos de importante mención, toda vez, que la actividad agrícola y pecuaria diferente a la ganadería extensiva se ha venido desarrollando de manera deficiente 
teniendo en cuenta que las políticas públicas locales, regionales y nacionales, claramente han preferido la explotación minera sobre la actividad propia que tiene que ver con la seguridad y soberanía alimentaria.

Es así como algunos subsectores como el arrocero vienen decreciendo en sus niveles de producción, y, por otro lado, sectores como la palma para producción de aceite, la caña y el maíz, se han convertido en actividades que desarrollan grandes inversionistas, muchas veces de otras partes del país o del extranjero que al final envían su producción a otras regiones, tales formas de producción culturalmente de tradición llanera ya no son del resorte de los campesinos locales.

Entonces, a pesar de que la seguridad y soberanía alimentaria aparece en el plan de desarrollo departamental y en las apuestas de diferentes instituciones de orden local y nacional, como una prioridad que hace parte del desarrollo integral del territorio, en la práctica no cuenta con herramientas suficientemente, contundentes e integrales para su fortalecimiento en los que se puede involucrar alternativas socioeconómicas y productivas como la agroecología, la agricultura familiar, los mercados locales o campesino y las economías solidarias, para reforzar la solución a las diferentes problemáticas que en este sentido aboca la reflexión en torno al tema.

De esta manera, este documento pretende reforzar una visión holística de la seguridad y soberanía alimentaria desde diferentes perspectivas, pero que si se integran en un análisis correcto establecerá un derrotero reflexivo para estimar las dinámicas que las realidades de los territorios presenten a razón de producción, cultura, economía, ambiente, organizaciones y sociedad.

\section{La sociedad centro de la seguridad y soberanía alimentaria.}

Vista desde el derecho, la alimentación es un pilar fundamental para el bienestar de la población, pues garantiza y democratiza la vida digna deseable para todos; para una nación en vía de desarrollo económico y humano reviste un asunto cardinal, por ende, la movilización de esfuerzos del estado y sociedad para proveer de alimentos inocuos, en las cantidades requeridas a la población, principalmente a aquellas comunidades que históricamente han estado al margen del desarrollo, debe ser contundente; entonces si se quiere, la seguridad alimentaria debe ser vista como asunto de primer orden nacional.

De igual manera, se debe considerar lo territorial para comprender las diferencias que caracterizan las regiones de Colombia, de las múltiples problemáticas y aspectos que inciden en los índices de medición de la desnutrición o la obesidad, por ejemplo. A propósito "De acuerdo con los hallazgos, el retraso en la talla de los niños menores de cinco años, si bien tuvo una mejora desde el 2010 al pasar del 13,2 por ciento a 10,8 por ciento, no logra todavía la meta establecida en los Objetivos de Desarrollo del Milenio (ODM) que había sido fijada en el 8 por ciento para el 2015". De igual forma, en el mismo grupo de edad se 
presentó un aumento de la desnutrición aguda (deficiencia de peso para la altura o delgadez extrema), al pasar del 0,9 al 2,3 por ciento; una situación contradictoria, según la misma encuesta, "si se tienen en cuenta los cambios positivos en la calidad de vida de la población colombiana en los últimos años".

Lo preocupante es que el aumento de la desnutrición aguda está relacionado, según los hallazgos, con el deterioro en la salud de los menores, al demostrar que el 47,3 por ciento de los afectados presentaba algún síntoma que indicaba problemas respiratorios 0 intestinales. De igual forma, al referenciar el indicador de desnutrición global en los primeros años de vida, se encuentra que está presente en casi cuatro de cada diez niños y que esta condición se duplica en los grupos indígenas, "lo que pone en evidencia una clara situación de inequidad y desigualdad social"

Si bien la edad escolar es una fase crucial en la que los menores experimentan un crecimiento continuo, es evidente que más del 7 por ciento de los niños entre 5 y 12 años del país presentan un retraso en la talla. Y esta situación, de acuerdo con los analistas, representa uno de los principales factores de transmisión intergeneracional de pobreza y desigualdad.

Al analizar a los adolescentes, se encontró que casi el 10 por ciento de ellos presenta retraso en la talla, con un patrón más marcado en los sitios con mayores inequidades, como las zonas indígenas y las zonas más alejadas del país." El tiempo, (2017)
Es importante observar estas cifras que alertan sobre la situación que se vive en el país, principalmente en la primera infancia y en general en la niñez, pues indican que, pese a los esfuerzos acometidos desde los gobiernos, no han sido suficientes para alcanzar las metas trazadas en el marco de organismos internacionales como la ONU. Es en la primera infancia donde se cimientan en el ser humano las características principales como su capacidad cognitiva, la talla, el peso, las capacidades esenciales para el buen desempeño.

Así mismo, algunas problemáticas y realidades en los territorios ahondan brechas y generan exclusión social; siendo muy diferente a su vez, el desarrollo en sectores urbanos y en sectores rurales; la dinámica del conflicto armado significó para el país un estancamiento en el desarrollo rural; la implementación de políticas públicas distanciadas de las necesidades sentidas de las comunidades campesinas, son entre otros, elementos estructurales que han ahondado la brecha territorial, que se traduce en aspectos como las deficientes vías secundarias y terciarias para el intercambio y comercialización de productos agrícolas y pecuarios, las debilidades para dinamizar un mercado nacional, la falta de competitividad de los campesinos en relación a empresas multinacionales en el marco de los TLC, la importación y contrabando de alimentos, son entre otros, problemas que afectan el poder garantizar la seguridad alimentaria para la población colombiana. Ahora bien, ante un panorama que exige plantear alternativas que mejoren significativamente el acceso 
social en cantidad y calidad de los alimentos que requiere la población es donde emergen perspectivas sostenibles y organizacionales, de agricultura limpia, de reutilización de saberes ancestrales y tradicionales puedan coadyuvar en este propósito.

La soberanía alimentaria hace parte de esta argumentación como forma legítima de producción tradicional de los campesinos, de las comunidades indígenas y afrodescendientes, en donde primeramente se busca proveer de alimentos para el autoconsumo familiar y comunitario, respetando con esas prácticas el medio ambiente, la tierra, el agua y así mismo promoviendo la articulación y el fortalecimiento de los tejidos sociales en prácticas como la minga, el trueque o la mano vuelta.

En la reciente gira realizada a finales del 2017 por investigadores de la Red de investigadores del Meta por diferentes departamentos de Colombia, se pudo apreciar, de una manera clara y elocuente por parte de las organizaciones y empresas que nos atendieron, la imperiosa necesidad de que en las formas de producir lo agrícola y lo pecuario se tengan en cuenta criterios de sostenibilidad y sustentabilidad, con el objeto de garantizar productos alimenticios de mayor calidad, de mejor aprovechamiento biológico, libres de químicos, respetando con mayor eficiencia los manejos de la tierra, las fuentes de agua, es decir, una vuelta al origen. Esta incorporación de técnicas artesanales, tradicionales, son a su vez un elemento que genera innovación, pues bajo este modelo se busca una maximización de los recursos naturales precisamente con el objetivo de aminorar costos de producción e impactos negativos en la explotación de los recursos, así como el fortalecimiento de redes campesinas de producción bajo nuevas formas de participación y asociatividad.

Ahora bien, profundizando un poco en el concepto de soberanía alimentaria se tiene que "Soberanía alimentaria implica la primacía de los derechos de los pueblos y las comunidades a la alimentación y la producción de alimentos, sobre los intereses del comercio. Por ello, en su propuesta antepone el fomento y la promoción de los mercados locales y de los productores frente a la producción para la exportación y la importación de alimentos". Es así, que, siguiendo esta línea argumentativa, la seguridad y soberanía alimentaria, en definitiva, aborda la temática no sólo del hambre, sino del desarrollo rural en general, desde un enfoque de derechos, que abarca desde el derecho a la alimentación, hasta la reivindicación del reconocimiento del derecho a producir y el derecho al acceso y la gestión de los recursos productivos, tales como tierra, agua, bosques, semillas, ganado y biodiversidad, para su uso sostenible". Rivera y Soler (s.f.)

Por otro lado, es necesario resaltar las experiencias de organizaciones con carácter asociativo como la de los mercados campesinos que se desarrollan en la actualidad en algunos municipios del Meta. Básicamente, estos espacios o mercados alternativos son la cristalización de procesos productivos, en general de manera orgánica y ecológica y que se encuentran inmersos dentro de la línea de construir escenarios idóneos para 
promover la seguridad y la soberanía alimentaria. Sin embargo, este tipo de iniciativas demandan un apoyo mucho más decidido por parte de los gobiernos locales, departamentales y nacional, así mismo del sector privado, en donde se impulsen redes de producción campesina (agricultura familiar) y se empiece a dar el salto cualitativo del consumo de alimentos con alta carga de productos químicos a alimentos más nutritivos, saludables, orgánicos.

Ahora bien, es necesario ser conscientes que el proceso no es fácil de implementar pues se está hablando de ciertos hábitos de consumo y patrones culturales-económicos arraigados y que estas propuestas alternativas van en contravía de los intereses de las grandes empresas nacionales y multinacionales que acaparan el mercado de alimentos en Colombia cuya única filosofía es la del costo beneficio financiero (crecimiento económico) y no la del costo beneficio social (desarrollo económico).

Por lo tanto, hablar de seguridad y soberanía alimentaria en nuestro país implica partir del reconocimiento de hallar caminos diferentes para atacar las desigualdades sociales, como se mencionó desde un enfoque de derechos y territorial y es pertinente dar el impulso estatal como factor estratégico de la nación, pues garantizar el acceso universal a alimentos adecuados en inocuidad y cantidad, bajo parámetros de sostenibilidad y de manejo limpio de los productos, indudablemente nos transformará en un país más competitivo, más innovador y equitativo apuntando a la mitigación de los efectos del calentamiento global.
Los cambios culturales son siempre procesos de largo cuño y para que se den debe existir una fuerte voluntad política y ciudadana con el ánimo de empezar a cambiar el paradigma respecto a las formas de producir los alimentos, propender por los precios justos y la economía solidaria y así mismo transformar hábitos en el consumo de los alimentos. No basta con proyectar e implementar políticas públicas, que si bien son importantes y se ajustan a los parámetros internacionales, es fundamental, desde esta perspectiva, realizar mayores esfuerzos institucionales y sociales para erradicar no solo la desnutrición sino el hambre de nuestro país, pues resulta impresentable, que con la riqueza que disponemos en Colombia, existan hoy, en el tercer lustro del siglo XXI, casos de muertes por desnutrición aguda en niños en diferentes regiones del país. En gran medida, esta idea se basa en la necesidad de revaluar el sistema económico y productivo, en el cual el extractivismo minero y energético, los monocultivos, así como la importación de alimentos, son entre otros factores problémicos que no permiten asegurar el autoabastecimiento de alimentos porque no están en simbiosis con otras formas y alternativas.

\section{La seguridad alimentaria como} determínate económico en el territorio e importancia estratégica desde la ciudadanía.

Cuando se menciona la perspectiva económica, inmediatamente el lector establece una conexión con temas financieros exclusivamente; causado seguramente por el hecho de que 
Colombia se fundamenta en criterios de corte capitalista, por lo que la educación y formación de sus ciudadanos se enmarca en el hecho de que la sostenibilidad del país esta soportada en una contabilidad de producción - consumo, de importaciones exportaciones es decir sobre el criterio de crecimiento económico ${ }^{1}$, dejando a un lado $\mathrm{o}$ en menor aprecio temas relacionados con el desarrollo económico ${ }^{2}$ que en general es el objetivo fundamental si se habla de dignidad, equidad e igualdad en un territorio.

Desde esta perspectiva la participación de sus ciudadanos de forma responsable es un hecho político y económico que no se adopta de la mejor manera, pues genera en muchos casos actuaciones de su parte poco o nada ajustadas a la necesidad real de cada territorio. Con manifestaciones de incoherencia que permiten diseñar políticas públicas desajustadas e ineficientes en la mayoría de los casos desgastantes.

De lo anterior y en el marco de este documento se establece dos aspectos relevantes a razón de participación en el territorio y el hecho de que existe una mala interpretación de la economía a nivel general, pero, sobre todo; en el impacto que esta tiene en la seguridad alimentaria.

Entonces, y en primera instancia el Ordenamiento Territorial $^{3}$ y más concretamente los Planes de Ordenamiento Territorial, son

\footnotetext{
1 En general el crecimiento económico esta relacionados con dinámicas como: Inflación, Desempleo, intereses, impuestos, aranceles e inversión, exportaciones e importaciones

2 En general relacionados con dinámicas de intervención: Gubernamental, ONG, Universidades, Organismos internacionales, regionales y locales se incluye aspectos relacionados con salud, vivienda, alimentación, recreación, seguridad, participación entre otras.
}

instrumentos que surgen como respuesta a desarrollos históricos de la sociedad colombiana, al responder ésta a tendencias y corrientes de pensamiento globales. Es exactamente la Constitución de 1991 y posteriormente la Ley 388 de 1997 o Ley de Desarrollo Territorial, que a su vez es una respuesta al Artículo 41 de la Ley 152 de $1994^{4}$, las que fortalecen y consolidan todos estos procesos de apertura democrática iniciados desde antes y donde se establecen nuevas relaciones y nuevos esquemas en la administración pública, en la relación entre gobernantes $y$ gobernados, en el marco de la concepción del Estado social de derecho; dándole a éste un carácter participativo por parte de la sociedad civil. Por esta razón se debe considerar de vital importancia, el diseño de un Plan a través de una metodología eminentemente participativa, investigativa y prospectiva, en la que los ciudadanos metenses, conforme lo exige nuestra Constitución, podrían en forma real intervenir en el ordenamiento de todo nuestro territorio.

Es importante ordenar nuestro territorio no solamente en su aspecto físico y espacial, sino que también es importante ordenar lo institucional, sociopolítico, ambiental, económico y por supuesto lo productivo. Entendido de esta manera, se manifestaría; como un reto a aceptar, con la certeza de que el desarrollo de los territorios beneficie a todos, así se tendrá otra actitud, otro comportamiento y otra

\footnotetext{
${ }^{3}$ Ley 388 de 1997

${ }^{4}$ Ley 152 de 1994 art. 41
} 
concepción ante el mundo local, regional y mundial. Esto tiene que ser así, porque el Territorio, como lo asume la Ley 388 y como se debe asumir, no sólo corresponde a lo físico, lo espacial, sino también a los aspectos sociales, políticos, culturales, económicos, productivos, ambientales y cultural $^{5}$. Y para poder cumplir con las directrices del ordenamiento territorial es necesario que los ciudadanos se involucren de forma responsable en algún momento y en alguna medida en la construcción de región.

Desde esta perspectiva, el dominio del ordenamiento territorial comprende un conjunto de acciones político administrativas y de planificación física "CONCERTADAS", emprendidas por los municipios, en "EJERCICIO DE LA FUNCIÓN PÚBLICA" que les compete, dentro de los límites fijados por la Constitución y las leyes, para disponer de instrumentos eficientes que orienten el desarrollo del territorio bajo su jurisdicción y regular la utilización, transformación y ocupación del espacio, de acuerdo con las estrategias de desarrollo socioeconómico y en armonía con el medio ambiente y las tradiciones históricas y culturales".

De hecho, el Ordenamiento Territorial municipal tiene por objeto complementar la planificación económica y social con la dimensión territorial, racionalizar las intervenciones sobre el territorio y orientar su desarrollo y aprovechamiento sostenible, mediante; la definición de estrategias territoriales de uso, ocupación y manejo del suelo, en función de los objetivos económicos, sociales, urbanísticos y ambientales. Es necesario el diseño y adopción de los instrumentos y procedimientos de gestión y actuaciones claras, que permitan ejecutar acciones urbanísticas integrales y poder articular las actuaciones sectoriales que afectan la estructura del territorio municipal; con la definición de los programas y proyectos que concreten estos propósitos.

Entonces el fin último y fundamental del Ordenamiento Territorial, es el logro de condiciones de vida dignas para la población actual y las poblaciones futuras; para ello es necesario proponer las estrategias metodológicas donde lo que impere es lo holístico y no lo especifico para ello se requiere, propiciar espacios eficientes donde bajo la convocatoria y las orientaciones técnicas y comunitarias, se reúnan todos los agentes sociales y los actores comunales, públicos y privados para reflexionar sobre la situación actual que el momento histórico amerite.

Ahora bien, en segunda medida y entorno a la seguridad y soberanía alimentaria es necesario entender que la misma es un elemento vital correspondiente al desarrollo económico de un territorio; en otras palabras, el estado a través del gobierno local o nacional debe orientar estrategias que establezcan el mantenimiento de la seguridad y soberanía alimentaria a la población en general para condicionar una sociedad sana, acta para ejecutar actividades que redunden en la sustentabilidad de este ciclo vital social. Sin embargo, es necesario precisar que

${ }^{5}$ Gabriel Espinosa Arrieta. Alcalde de Sincé (Sucre). 1998 
para el caso existen dos tipos de economía en este marco; con filosofías y objetivos específicos diferentes pero que en la práctica son aportantes en la sustentabilidad del ciclo vital social mencionado.

La economía campesina y la economía agropecuaria primas hermanas que tomaron caminos diferendos desde la perspectiva de sus modos de producción, pero con similitud desde el punto de vista de los actores involucrados (campesinos). Adicionalmente se entre ve elementos de participación diferenciados con pensamientos políticos que arraigan su proceder los cuales se esbozan a continuación.

La economía campesina se fundamenta sobre una concepción política socialista, es decir; sobre la actuación asociativa y cooperativa de sus actores, toda la familia interviene como agente de producción, se desarrollan policultivos en muchos casos de carácter mixto (agrícola $\mathrm{y}$ pecuario), se genera excedentes, las áreas de producción promedio son de 1 a $10 \mathrm{Ha}$, económicamente se presentan diferentes niveles de subsistencia, transicional y excedentario, es una actividad potenciada por la organización y cooperación de muchas familias, los niños $\mathrm{y}$ niñas intervienen activamente lo que permite el relevo generacional y es un sistema ambientalmente sostenible y sustentable

En contra posición la economía agropecuaria es de corte capitalista, la actuación de sus actores son individuales, la familia en si no interviene en el modo de producción, se desarrolla de forma intensiva monocultivos, desde un punto de vista financiero se busca la generación de rentabilidad, las áreas de intervención en promedio son mayores de a $20 \mathrm{Ha}$, en la mayoría de los casos se genera producción a escala, es una actividad potenciada por un individuos o socios en ella los niños y niñas no intervienen, normalmente no hay relevo generacional, ambientalmente es poco sustentable frente al medio ambiente.

Sin embargo y se ha demostrado a través de la historia de los pueblos que ninguna de las dos es suficiente para abastecer la necesidad de alimento para las poblaciones, sino que se necesita de las dos para mantener el ciclo vital social, es decir; ninguna es la verdad revelada ni el modelo justo para mantener índices de igualdad y equidad.

Lo anterior, se enuncia teniendo en cuenta que la economía campesina complementa los vacíos que a razón de alimentos se genera o que no es capaz de suplir la economía agropecuaria por lo que la seguridad alimentaria del territorio se equilibrará. Y en otro sentido la economía agropecuaria complementa los vacíos que a razón de empleo genera y que no es capaz de suplir la economía campesina por lo que se regulará el empleo en el territorio.

En general y solo con estas dos menciones se ordena el territorio de forma participativa privilegiando el empleo y la seguridad alimentaria al mismo tiempo de lo cual se genera como resultado fortalecimiento de un desarrollo y crecimiento económico de forma simbiótica. 
Semillas, usos y saberes como estrategia para el fortalecimiento de la seguridad y soberanía alimentaria en la Orinoquia colombiana.

Las semillas han sido mantenidas y seleccionadas mediante el trabajo, la experimentación y el cuidado colectivo en los pueblos y, a su vez, ellas han hecho parte importante del desarrollo de las culturas ancestrales y han servido para transmitir los conocimientos adquiridos por las comunidades indígenas $\mathrm{y}$ campesinas a través de todo el mundo. Asimismo, se integran en un proceso constante de creación y renovación.

Las semillas y las razas de los animales criollas y nativas son una fuente de bienestar para las comunidades y representan la mejor posibilidad para mejorar la calidad de vida y asegurar la conservación de los ecosistemas silvestres, así como el mantenimiento y protección de los suelos, las fuentes de aguas, los sistemas productivos agroecológicos locales, la alimentación y los saberes tradicionales de las comunidades. Las semillas criollas son fundamentales para garantizar la soberanía y autonomía alimentaria de los pueblos y son el seguro para enfrentar la crisis alimentaria y salvaguardar la agricultura frente al cambio climático; es así como la diversidad de semillas criollas y los sistemas de producción tradicionales, se constituyen en una estrategia de resiliencia indispensables frente al cambio climático.

Las semillas criollas también son el mejor instrumento que tienen las comunidades rurales para enfrentar el modelo de desarrollo agrícola insostenible, basado en monocultivos agroindustriales que imponen la dependencia a las semillas híbridas y transgénicas, las cuales han sido patentadas y controladas por las empresas semilleros que pretenden controlar todo el sistema agroalimentario.

Es preocupante la progresiva pérdida de semillas criollas (tanto silvestres como cultivadas) y conocimientos tradicionales de las comunidades indígenas, afro y campesinas, lo que ha generado la perdida de la soberanía y autonomía alimentaria de las comunidades locales. Algunas de las principales causas de la perdida de la agrobiodiversidad son: la destrucción de los bosques y los ecosistemas naturales; la perdida de los conocimientos tradicionales y de los sistemas de producción locales; las presiones demográficas; el pastoreo excesivo; los monocultivos agroindustriales; los proyectos mineroenergéticos; los cambios en las prácticas agrícolas; y el control monopólico de las semillas por las empresas.

Ante la necesidad de recomponer los sistemas productivos tradicionales, con la utilización de las semillas y razas criollas se buscan mejores condiciones de calidad de vida, que permitan la permanencia en el campo en condiciones dignas y el reconocimiento de los saberes y el trabajo de los mayores y especialmente de las mujeres.

La libre circulación de las semillas entre comunidades y pueblos es sin duda la mejor manera de recuperar y usar las semillas. Para ello han sido fundamentales las múltiples estrategias y acciones que han implementado las comunidades 
rurales desde épocas ancestrales, como son los trueques, los intercambios, los mercados campesinos y/o comunitarios, los encuentros de sabedores, entre otros, donde se comparten las semillas, los saberes y sabores de la comida tradicional, que en su conjunto cumplen la función de garantizar la soberanía y autonomía alimentaria de los pueblos y comunidades.

\section{Conclusiones}

La seguridad alimentaria es una cuestión superior en la construcción de país, no es solo una política pública estatal, ni una medida de cumplimiento a parámetros deseables internacionalmente, es, la piedra angular de garantía de goce efectivo de derechos para la población, en particular para la primera infancia y para las comunidades más rezagadas de los beneficios del sistema socioeconómico imperante. Por consiguiente, es imprescindible superar modelos y paradigmas de producción agropecuaria anacrónicos y dar el salto cualitativo a nuevas formas, que, en últimas, corresponden a una vuelta al origen, por ejemplo, el hecho de producir amigablemente con los ecosistemas, de manera limpia, bajo modelos de economía familiar y campesina que promueva y fortalezca nuevas formas de asociatividad rural y nuevas formas de reciprocidad y equidad en el desarrollo urbano-rural.

En el crisol cultural y ambiental que es Colombia, es fundamental pensar la soberanía alimentaria como una poderosa herramienta de desarrollo económico y progreso humano, es decir, de transformación. Es muy importante hallar caminos alternativos al tradicional modelo capitalista y a los lineamientos del neoliberalismo económico, que se caracterizan, entre otras cosas, por ser altamente excluyentes socialmente hablando y que atentan contra la armonía de los ecosistemas de la nación.

Desde esta perspectiva cuando es mencionada el componente económico, inmediatamente se establece una conexión financiera exclusivamente; enmarca en el hecho de que la sostenibilidad del país esta soportada en una contabilidad de producción - consumo, de importaciones exportaciones es decir sobre el criterio de crecimiento económico.

Es así como el documento establece aspectos relevantes a razón de participación en el territorio y el hecho de que existe una mala interpretación de la economía a nivel general, pero, sobre todo; en el impacto que esta tiene en la seguridad alimentaria.

Entonces se plantea la necesidad de ordenar el territorio de forma participativa y responsable anotando en torno a la seguridad y soberanía alimentaria, conceptos fundamentales y de vital importancia que generen claridad en la toma de decisiones de las poblaciones en los territorios; como lo es la economía campesina y la economía agropecuaria. Haciendo hincapié en que la "economía campesina complementa los vacíos que a razón de alimentos se genera o que no es capaz de suplir la economía agropecuaria por lo que la seguridad alimentaria del territorio se equilibrará. Y en otro sentido la economía agropecuaria complementa los vacíos que a razón de empleo genera y 
que no es capaz de suplir la economía campesina por lo que se regulará el empleo en el territorio".

De hecho, se resalta como resultado el fortalecimiento de un desarrollo y crecimiento económico de forma simbiótica en el territorio de forma local desde sus actores directos.

Así las cosas, la sociedad, las comunidades, la cultura, la economía y el sector productivo, deben crear sinergias que realmente beneficien la Seguridad y Soberanía alimentaria, puesto que, entendiendo los retos del futuro como el cambio climático, el aumento exponencial de la población mundial, el deterioro de los recursos naturales y la dinámica económica y financiera que las grandes potencias mundiales proponen, será decisión de los pueblos y sus gobernantes, determinar las estrategias adecuadas para lograr la supervivencia de sus comunidades, su cultura y sus territorios.

\section{Referencias}

El tiempo (2017) "Sedentarismo y mala alimentación dispararon el sobrepeso y la obesidad". El tiempo, publicado 22 de noviembre de 2017. Tomado de http://www.eltiempo.com/vida/salud/cifra s-de-la-obesidad-y-la-desnutricion-encolombia-153944

Rivera y Soler (s.f.) "El enfoque de la soberanía alimentaria: más allá de la seguridad alimentaria" grupo de investigación ARAG Universidad Autónoma de Barcelona. Tomado de http://www.fes- sociologia.com/files/congress/10/grupostrabajo/ponencias/892.pdf.

Pacheco, C. (2016). Impacto económico de la violencia armada sobre la producción campesina, caso municipios zona de distensión departamento del Meta, Colombia (1991-2014). Revista Lebret, 8. Bucaramanga, Colombia: Universidad Santo Tomás, pp. 93 - 123. ISSN: 21455996

Espitia, L., Pacheco Pérez, C., \& Guerrero Moreno, S. (2017). Construcción de un modelo de desarrollo sostenible para el municipio de Lejanías - Meta de manera concertada con los actores territoriales para la vigencia 2016 - 2019. Revista GEON (Gestión, Organizaciones Y Negocios), 4(2), 101-115. Recuperado a partir

de

http://revistageon.unillanos.edu.co/index. $\mathrm{php/geon/article/view/28}$

Bielschowsky, R. (2010). Estrategia de desarrollo y política industrial en Brasil. Retrieved July, 20, 2012.

Pedraza, D. F. (2004). Estado nutricional como factor y resultado de la seguridad alimentaria y nutricional y sus representaciones en Brasil. Revista de Salud Pública, 6(2), 140-155

Ley 388 (1997) Republica de Colombia. Ordenamiento territorial

Ley 152 (1994) Republica de Colombia. Planes de desarrollo.

Red de Semillas Libres de Colombia (RSL) (2013.) Memorias del Primer Encuentro Nacional. Bogotá D.C.

Grupo Semillas. Guía metodológica para el diagnóstico, recuperación, 
conservación y difusión de semillas criollas (2016). Arfo Editores. 44p.

Cruz, N.E., Cortes, J.E., Vélez, N., Fagua, J.S., Pineda, E.O., González, G., Guantiva, E.O., Gómez, E \& Vargas, O.J. 2015. Protección del conocimiento ancestral en recursos florísticos para mejoramiento de la calidad de vida de comunidades indígenas del Meta. Corporación Universitaria Minuto de Dios. 